\title{
DIE SEGMENTIERUNG DES CRUSTACEEN-KOPFES UND DER CEPHALOTHORAX DER DECAPODEN
}

\author{
VON \\ J. E. W. IHLE UND M. E. IHLE-LANDENBERG \\ (Zoologisches Institut der Universitat Amsterdam)
}

Als wir gefragt wurden einen Beitrage für diese Festschrift zu liefern, haben wir dies gern getan und sind also jetzt in der Lage dem Vorstand und dem tüchtigen Direktor der königlich zoologischen Gesellschaft unsere herzlichen Glückwünsche anzubieten zum 100 . Geburtstag der Gesellschaft. Wir hoffen, dass sie nach Überwindung der Schwierigkeiten ein zweites ruhmvolles Jahrhundert erleben wird. Ebenso geben wird der Hoffnung Ausdruck, dass der zoologische Unterricht durch die Lage des zoologischen Instituts im zoologischen Garten und durch das angenehme Verhältnis der Gesellschaft zum Institut fortwährend gefördert werden wird.

In diesem kurzen Aufsatz wollen wir auseinandersetzen, wie neuere Ansichten über die Segmentierung des Crustaceen-Kopfes Einfluss haben auf unsere Auffassungen des Cephalothorax der Decapoden und zugleich einige Merkmale des Cephalothorax dieser Tiere erörtern.

Aus den Untersuchungen von Holmgren und Hanström hat sich ergeben, dass das Proto- und Deuterocerebrum des Gehirnganglions der Crustaceen, die resp. Augen und Antennae I innervieren, primär präoral gelegene Hirnteilę. sind mit präoralen Commissuren. Phylogenetisch sind sie durch sekundäre Teilung des Cerebralganglions (= Archicerebrum) der Anneliden, das im Prostomium liegt, entstanden. Holmgren bestätigte die Auffassung, dass die Antennen I der Crustaceen mit den Palpen der Polychaeten vergleichbar sind, wie die Palpenglomeruli der Polychaeten den Antennalglomeruli der Crustaceen homolog sind. In beiden Gruppen doch zeigen diese Glomeruli dasselbe Verhalten zu den Corpora pedunculata, den wichtigsten Assoziationszentra im Archicerebrum.

Aus der Tatsache, dass Augenstiele und Antennen I von primär präoralen Hirnteilen innerviert werden, dürfen wir schliessen, dass diese Anhänge den übrigen (postoralen) Gliedmassen nicht homodynam sind. Dies stimmt durchaus mit der bekannten Tatsache, dass die Antennen 1 1) der Crustaceen im

r) Wir ziehen es vor die Namen I. und 2. Antenne zu gebrauchen statt Antennula und Antenne, da nur die I. Antennen den Antennen der Tracheaten homolog sind und also bei der Benutzung der Namen Antennula und Antenne die Antennulae der Crustaccen den Antennen der Tracheaten homolog sind und die Antennen der Crustaceen bei den Tracheaten fehlen. Sie sind den Cheliceren der Arachnoidea vergleichbar. 
Gegensatz zu den übrigen Extremitäten schon bei ihrer ersten Entstehung keine Spaltfüsse sind, wodurch sie ihren prinzipiellen Unterschied den typischen Extrèmitäten gegenüber zeigen. Die Augenstiele besitzen übrigens übérhaupt keine grössere Ähnlichkeit mit Gliedmassen.

Die Auffassung, dass Augenstiele und Antennen I der Crustaceen zum Prostomium gehören, wurde ausser von Holmgren und Hanström u. A. von Balls (I93I), Sollaud (1933), Snodgrass (I935) und H. Weber (I937, p. I I3) verteidigt.

Die 2. Antenne der Crustaceen ist also die 1. postorale Extremität. Dies geht auch hieraus hervor, dass sie wie die Cheliceren der Arachnoidea vom Tritocerebrum innerviert wird, dem ursprünglich postoral gelegenen, vorderen Ganglion der Bauchganglienkette. (mit einem postoralen Kommissur), das selbst bei den Phyllopoden selbständig bleibt und hier nicht mit dem eigentlichen Hirnganglion (Archicerrebrum) zum Syncerebrum verschmilzt.

Wenn man diese Ansicht teilt, können die Cölomsäckchen im präoralen Teil des Kopfes von manchen Arthropoden nicht als Zeichen für das Vorhandensein von Segmenten gelten, die ursprünglich postoral gelegen, sekundär eine präorale Lage bekommen haben. So hat z. B. Heymons (1901) bei Scolopendra Cölomsäckchen beschrieben im Präantennen-, Antennen- und Prämandibular- (Intercalar-) Segment. Von diesen Säckchen können die im Präantennen- und Antennensegment gelegenen nicht den übrigen homodynam sein, und müssen als sekundär erworben betrachtet werden. Das Prämandibularsegment ist nach der hier verteidigten Auffassung das 1. postorale Segment und dem Segment der 2. Antenne der Crustaceen vergleichbar.

Der vordere Teil des Cephalothorax der Crustaceen mit Augenstielen und Antennae I; der dem Prostomium der Anneliden entspricht, wollen wir V o r d e r k o p f nennen. Er weicht also prinzipiell vom postoralen, segmentierten Teil des Cephalothorax ab. Bei Penaeus und Palaemon ist dieser Vorderkopf gelenkig, also beweglich mit dem übrigen Teil des Cephalothorax verbunden (K. GrobbeN I9I7, p. 487). Er hat hier also eine grössere Selbständigkeit dem übrigen Teil des Cephalothorax gegenüber. Auch bei den Stomatopoden ist der Vorderkopf beweglich geworden, aber in 2 'Teile zerfallen ("Augensegment" und "Antennularsegment”). Andererseits können auch Teile des mit dem übrigen Teil des Cephalothorax verwachsenen Vorderkopfes selbständig werden, wie der Augenbogen bei manchèn Galatheiden und Paguriden nur durch weiche Häute mit seiner Umgebung verbunden ist.

Wir bemerken noch, dass der Name primäres Kopfsegment, der. wohl für den Vorderkopf der Crustaceen gebraucht wird, keine Empfehlung verdient, da dieser Körperteil nicht den postoralen Segmenten homodynam ist.

Fragen wir jetzt, inwieweit die neue Auffassung über die Segmentierung des Kopfes Einfluss hat auf die Betrachtung des Cephalothorax der Decapoden. Wir heben aber erst hervor, dass jetzt keine gründliche Betrachtung der 
Morphologie dieses Körperteils folgt; nur einige Bemerkungen werden von uns gemacht.

Wenn der Vorderkopf mit dem Prostomium der Anneliden vergleichbar ist, kann man in dieser Körperabteilung selbstverständlich nicht die Teile der postoralen Segmente (Tergit, Sternit) zurückfinden. Der Augenbogen, auf welchen die Augenstiele eingeplanzt sind, ist also kein Sternit und ebensowenig gehört die Region, wo die Antennae I eingepflanzt sind, zu einem Sterniten.

Der A ug e nbogen (l'arceau ophthalmique von A. Milne Édwards und Bouvier, der Querzylinder von NeTz) ist ein querer Chitinbalken, der zum ventralen Teil des Vorderkopfes gehört. Ursprünglich schmal, wird er in manchen Fällen, wo die Augen weit von einander entfernt sind, breit wie bei den Palinuriden, Scyllariden und vielen Brachyuren. Bei letztgenannten verbindet das Rostrum sich median mit dem Epistom (CALMAN 1909, p. 257) und der Augenbogen ist von aussen nicht mehr sichtbar.

Die R e gio n, wo die A.n te n n a e I eingepflanzt sind, ist nicht von der Umgebung abgegrenzt und geht also nach hinten in das Epistom über.

Das E p is tom wird wohl mit Recht als das Sternit des Segments der 2. Antenne aufgefasst (CALman 1909, p. 257), obwohl auch andere Teile am Aufbau des Epistoms beteiligt sein können. Es stellt also das I. postorale Sternit dar, das bei der Bildung des Kopfes eine präorale Lage bekommen hat.

Über den $\mathrm{C}$ a $\mathrm{p}$ a $\mathrm{r} \mathbf{a} \mathrm{x}$ bemerken wir noch folgendes. Sehr oft wird dieser Name ganz unrichtig als synonym gebraucht für Cephalothorax, aber wir weisen nachdrücklich darauf hin, dass der Carapax eine Falte ist, die vom Hintenund Seitenrand des Kopfes ausgeht, hinten und median mit den 'Thoraxsegmenten verwächst und seitlich als Branchiostegit die Kiemen bedeckt. Wir bemerken noch, dass die vordere ventrale Ecke des Carapax mit dem Epistom verwachsen kann, wei bei den Scyllariden und Brachyuren (CalmaN, 1909, p. 257). Bei letztgenannten findet man manchmal eine Naht auf der Grenze zwischen Epistom und Carapax.

Es würde eine lohnende Aufgabe sein die $\mathrm{Sta}$ cheln des Cephalothorax in den verschiedenen Decapoden-Gruppen vergleichend zu studieren, da die einander in der Lage entsprechenden Stacheln oft wenigstens homoiolog sind. Wir erwähnen aber nur den Anterolateralstachel, der bei vielen Arten an der vorderen Aussenecke des Cephalothorax auftritt und dem Postoculardorn entspricht (Ihle und Ihle-Landenberg 1931, p. I48). Dieser Dorn kam vielleicht auch bei den Stammformen der Decapoden vor, da er bei Anthrapalaemon aus dem Karbon vorhanden ist. Diese Form ist nach BeurLen (I930, p. 442) vielleicht die dorsale Seite von Pygocephalus, der als Stammform der Decapoden betrachtet wird. 


\section{LITERATUR}

Balss, H. 193I. Crustacea, in: Handwörterbuch der Naturwissenschaften. 2. Aufl. Bd. 2. Beurlen, K. 1930. Vergleichende Stammesgeschichte. Grundlagen, Methoden, Probleme unter besonderer Berücksichtigung der höheren Krebse. Fortsch. Geol. Palaeont. Bd. 8, Heft 26.

Calman, W. T. Crustacea, in Ray Lankester: A treatise on zoology. Pt. 7.

Grobren, K. 1917. Der Schalenschliessmuskel der dekapoden Crustaceen, zugleich ein Beitrag zur Kenntnis ihrer Kopfmuskulatur. Sitzungsber. K. Akad. Wien. Abt. I. Bd. 126.

Ihle, J. E. W, und Ihle-Landenberg, M. E. I931. U'ber einige Tiefsee-Brachyuren der Siboga-Expedition aus der Gruppe der Oxyrhyncha. Zool. Anzeiger Bd. 93.

Snovgrass, R. E. 1935. Principles of insect morphology. New York and London.

Sollaud, E. 1933. Le blastopore et la question du „prostomium” chez les Crustacés. Association française pour l'avancement des sciences. C. R. de la $57 \mathrm{e}$ session: Chambéry.

WraER, H. 1937. Morphologie und Entwicklungsgeschichte der Arthropoden, in: Fortschr. der Zoologie. N.F. Bd. I.

\section{NACHSCHRIFT -}

Nachdem obenstehender Aufsatz schon längst im Druck war, wurden wir durch den 3. Band der Fortschritte der Zoologie, N. F. Bericht über das Jahr 1937 (H. Weber, Morphologie und Entwicklungsgeschichte der Arthropoden) bekannt mit der Abhandlung von K. FAHLANDER, Beitrag zur Anatomie und systematische Einteilung der Chilopoden, Zoologiska Bidrag från Uppsala, Bd. 17. Die Abhandlung selbst haben wir indessen noch nicht einsehen können.

Fahlander lehnt die Homologie zwischen den I. Antennen und den Palpen der Anneliden $a b$ und betrachtet die I. Antennen als die Gliedmasse des I. Kopfsegments. Der Kopf der Crustaceen besteht nach ihm also aus Acron (Prostomium) und 5 Segmenten; ein Präantennalsegment fehlt.

Wir bemerken, dass nach dieser Auffassung der in mancher Hinsicht auffällige Gegensatz zwischen I. und 2. Antenne unaufgeklärt bleibt, der begreiflich wird, wenn man die 1. Antennen nicht für homodynam mit den postoralen Extremitäten hält. Auch bei den Trilobiten und ihren Verwandten besteht ein schroffer Gegensatz zwischen den Antennen und allen übrigen Gliedmassen.

Schliesslich möchten wir z. B. noch auf die Abhandlung E. Sollaud's weisen (Recherches sur l'embryogénie des Crustacés décapodes de la sousfamille des Palaemoninae, Bull. biol. France Belgique. Suppl. 5, 1923, p. 103 und Pl. I, fig. 7), der bei Embryonen von Leander eine deutliche Furche hinter dem Prostomium sieht und wahrnimmt, dass auf dem Prostomium jederseits Augenanlage und die Anlage der I. Antenne aus einer gemeinsamen Anlage hervorgehen und dies gilt auch für die Ánlagen von Proto- und Deuterocerebrum. 